\title{
N-Acetylglucosamine Catabolism: Unique Side of Sugar Sensing
}

\section{Asis Datta* \\ National Institute of Plant Genome Research (NIPGR), Aruna Asaf Ali Marg, JNU Campus, New Delhi - 110067, India}

*Corresponding author: Asis Datta, Distinguished Scientist, National Institute of Plant Genome Research (NIPGR), Aruna Asaf Ali Marg, JNU Campus, New Delhi 110067, India, Tel: 91-11-26742750, 26735119; Fax: 91-11-26741759; E-mail: asis_datta@rediffmail.com, asis_datta@nipgr.ac.in

Rec date: June 17, 2015; Acc Date: June 23, 2015; Pub date: June 25, 2015

Copyright: $\odot 2015$ Datta S. This is an open-access article distributed under the terms of the Creative Commons Attribution License, which permits unrestricted use, distribution, and reproduction in any medium, provided the original author and source are credited.

\section{Commentary}

The success of any pathogenic microorganism lies in its ability to adapt to diverse and often stressful conditions within the host. For this, pathogens have developed myriad ways of parallel metabolic pathways, complex regulatory systems and stress adaptive mechanisms, which are best suited to the variety of environmental conditions they encounter within the human host.

$\mathrm{N}$-acetylglucosamine (GlcNAc) is among the most abundant sugars on the planet. This amino sugar is also well known for the important structural roles that it plays at the cell surface. It is a key component of bacterial cell wall peptidoglycan, fungal cell wall chitin, and the extracellular matrix of animal cells. GlcNAc is present only in trace amounts in plants, in contrast to the huge amount of various sugars that compose the polysaccharides of the plant cell wall. Interestingly, recent studies have also identified new roles for GlcNAc in cell signaling. For example, GlcNAc stimulates the human fungal pathogen Candida albicans to undergo changes in morphogenesis and expression of virulence genes. Pathogenic E. coli responds to GlcNAc by altering the expression of fimbriae and CURLI fibers that promote biofilm formation and GlcNAc stimulates soil bacteria to undergo changes in morphogenesis and production of antibiotics. Studies with animal cells have revealed that GlcNAc influences cell signaling through the post-translational modification of proteins by glycosylation. Moreover, several pathogens including C. albicans, Vibrio cholerae, Leishmania major, Peudomonas, aeruginosa utilize GlcNAc as a sole carbon source. GlcNAc metabolic pathway mutants of these pathogens are avirulent. Thus, GlcNAc also serves as in-vivo carbon source for pathogens. Within the host $C$. albicans probably encounters GlcNAc in most of the natural environments it inhabits, especially the mucosal layers.

Despite its central role as a nutrient molecule GlcNAc was never considered as a putative substrate that could be exploited by pathogenic/phytopathogenic microorganisms during infection. Our literature search, combined with bioinformatic analyses and experimental approaches show that $\mathrm{N}$-acetylglucosamine utilization pathway is quite common among other pathogenic microorganisms too. The enzymes needed to catabolize GlcNAc include Ngtl (a transporter that takes up GlcNAc), Hxk1 (a kinase that converts GlcNAc to GlcNAc-6-phosphate), Dac1 (a deacetylase that converts GlcNAc-6-phosphate to glucosamine-6-phosphate), and Nag1 (a deaminase that converts glucosamine-6-phosphate to glucose-6phosphate) [1]. In prokaryotic systems the enzymes needed for catabolism are almost similar except for the fact that sometimes a single gene serves the function for two catabolic steps. In Candida albicans, GlcNAc can regulate the expression of genes involved in metabolism [2-4], morphogenesis [5] switching [6] and virulence [7].
Although there are reports in the literature about the utilization of chitin and sialic acid by $V$. cholerae [8,9], the significance of $V$. cholerae's ability to intracellularly catabolize GlcNAc monomer, which is centrally important for all converging amino sugar utilization is still not fully understood. Our work with pathogenic microorganisms Vibrio cholerae show GlcNAc utilization to be an important aspect for the pathogen to reach sufficient titres in the intestine for producing clinical symptoms of cholera [10]. In other pathogenic organisms also this $\mathrm{N}$-acetylglucosamine utilization seemed to be very common. For example, it was seen that a mutant of Candida albicans, a fungal pathogen, incapable of utilizing GlcNAc was avirulent in a murine model of systemic candidiasis [7]. Studies on two species of dental plaque forming bacteria, Streptococcus mutans and Streptococcus sobrinus have shown that even though $S$. sobrinus is more acidogenic than $S$. mutans, it is less frequently isolated from human population as it is incapable of utilizing GlcNAc [11,12]. This further suggests that the organisms capable of utilizing GlcNAc as carbohydrate source are better adapted to infect and persist inside the host. In Gram-negative opportunistic pathogen Bacteroides fragilis, N-acetyl-D-glucosamine has been reported to be an excellent carbon and energy source and can be utilized more efficiently than glucose. In the human pathogens Streptococcus pneumoniae and Streptococcus oralis, monosaccharides released during the sequential degradation of complex-type N-glycans from human glycoconjugates are also used as a source of fermentable carbon.

GlcNAc has also never been considered a substrate exploited by phytopathogenic bacteria during plant infection. A recent study by Boulanger et al. [13] shows that Xanthomonas campestris pv. campestris, the causal agent of black rot disease of Brassica plants, expresses a carbohydrate utilization system devoted to GlcNAc exploitation and metabolizes GlcNAc in planta and in vitro. Further work in our lab is aimed to characterise the GlcNAc utilization pathway in other pathogens like Leishmania, Mycobacterium and plant pathogen like Magnaporthe grisea. Initial observations with GlcNAc catabolic pathway mutants show that the mutants are severely compromised in establishing infection. Therefore, these results and observations from our group and others significantly point out towards a universal role of the GlcNAc catabolic pathway in establishing infection among microorganisms.

\section{References}

1. Natarajan K, Datta A (1993) Molecular cloning and analysis of the NAG1 cDNA coding for glucosamine-6-phosphate deaminase from Candida albicans. J Biol Chem 268(13): 9206-9214.

2. Biswas M, Singh N, Datta A (1979) Inductionof N-acetylglucosamine catabolic pathway in yeast. Biochim Biophys Acta 585: 535-542.

3. Kamthan M, Kamthan A, Ruhela D, Maiti P, Bhavesh NS, et al. (2013) Upregulation of galactose metabolic pathway by $\mathrm{N}$-acetylglucosamine 
Citation: Datta A (2015) N-Acetylglucosamine Catabolism: Unique Side of Sugar Sensing. Biochem Anal Biochem 4: 189. doi: 10.4172/2161-1009.1000189

Page 2 of 2

induced endogenous synthesis of galactose in Candida albicans. Fungal Genet Biol 54: 15-24.

4. Ghosh S, Rao KH, Bhavesh NS, Das G, Dwivedi VP, Datta A (2014) NAcetylglucosamine (GlcNAc)-Inducible geneGIG2 Is a Novel Component of GlcNAc Metabolism in Candida albicans. Eukaryotic cell 13: 66-76

5. Shepherd MG and Sullivan PA (1983) Candida albicans germ-tube formation with immobilized GlcNAc. FEMS Microbiol Lett 17: 167-170

6. Huang G, Yi S, Sahni N, Daniels KJ, Srikantha T, Soll DR (2010) Nacetylglucosamine induces white to opaque switching, a mating prerequisite in Candida albicans. PLoS Pathog 6(3): e1000806.

7. Singh P, Ghosh S, Datta A (2001) Attenuation of virulence and changes in morphology in Candida albicans by disruption of the $\mathrm{N}$-acetylglucosamine catabolic pathway. Infect Immun 69(12): 7898-7903.

8. Meibom KL, Li XB, Nielsen AT, Wu CY, Roseman S, et al. (2004) The Vibrio cholerae chitin utilization program. Proc Natl Acad Sci U S A 101(8):2524-2529.
9. Almagro-Moreno S and Boyd EF (2009) Sialic Acid Catabolism Confers a Competitive Advantage to Pathogenic Vibrio cholerae in the Mouse Intestine. Infect Immun 77(9): 3807-3816.

10. Ghosh S, Rao KH, Sengupta M, Bhattacharya SK, Datta A (2011) Two gene clusters co-ordinate for a functional $\mathrm{N}$-acetylglucosamine catabolic pathway in Vibrio cholerae. Mol Microbiol. 80(6):1549-1560.

11. Homer KA, Patel R, Beighton D (1993) Effects of N-acetylglucosamine on carbohydrate fermentation by Streptococcus mutans NCTC 10449 and Streptococcus sobrinus SL-1. Infect Immun 61: 295-302.

12. Singh B and Datta A (1979) Inductionof N-acetylglucosamine catabolic pathay in spheroplast of Candida albicans. J biochmem 178: 427-431

13. Boulanger A, Zischek C, Lautier M, Jamet S, Rival P, et al. (2014) The plant pathogen Xanthomonas campestris pv. campestris exploits Nacetylglucosamine during infection. MBio 9: (5)e01527-1514. 